\title{
Are red blood cell transfusions associated with nosocomial infections in critically ill children?
}

\author{
Omar E. Naveda Romero, M.D. ${ }^{a}$ and Andrea F. Naveda Meléndez, student ${ }^{a}$
}

\begin{abstract}
Although the transfusion of blood products is common practice, its effects on the immune system have not been adequately studied. A prospective cohort study was conducted in critically ill children followed up until their death, transfer or discharge to establish an association between red blood cell transfusions (RBCTs) and nosocomial infections. A nosocomial infection was considered to be post-transfusional if it occurred within 14 days after RBCT. A total of 162 children were included in the study, $35(21.6 \%)$ had a nosocomial infection, and $49(30.2 \%)$ received a RBCT. Among those with a nosocomial infection, a RBCT was more common ( $48.5 \%$ versus $14.9 \%$, OR: 5.4 , 95\% CI: $2.4-$ $12.6, p<0.0001)$ and mortality rate was higher $(45.7 \%$ versus 10.2\%, OR: $7.4,95 \%$ CI: 3.1-18.2, $p<0.0001$ ). The binary logistic regression showed that RBCT was independently associated with nosocomial infections (OR: 4.2,95\% CI: 2.1-20.2, $p=0.049$ ). Conclusion: RBCT was associated with increased risk for nosocomial infections.

Key words: blood transfusion, red blood cells, nosocomial infections, children.
\end{abstract}

http:/ /dx.doi.org/10.5546/aap.2016.eng.347

\section{INTRODUCTION}

Red blood cell transfusions (RBCTs) are common practice in the management of critically ill children. In spite of the high volume of blood products administered in trauma, surgery and pediatric intensive care units, the effects of blood transfusions on the immune system have not been adequately studied. ${ }^{1}$

Over the past century, RBCTs have been considered a simple procedure with a clear benefit. However, this practice has been under close scrutiny. RBCTs have been recognized to

\footnotetext{
a. Department of Shock Trauma and Stabilization. Hospital Universitario de Pediatría "Dr. Agustín Zubillaga". Barquisimeto. Lara State. Venezuela.
}

E-mail address: Omar E. Naveda Romero, M.D. omarnavedamd@yahoo.com

Funding: None.

Conflict of interest: None.

Received: 9-6-2015

Accepted: 1-28-2016 pose risks associated with storage and in relation to the immunomodulatory effects that occur in almost all recipients; this underlines a possible association between RBCT and higher mortality and nosocomial infection rates..$^{2-4}$

Recognizing these risks has resulted in a more critical approach to the benefits associated with RBCT. The underlying mechanisms of immune response modulation by RBCT may include biological response modifiers of white blood cells, impaired T cell and natural killer cell activity, and defects in antigen presentation. ${ }^{5}$

The objective of this study was to establish a possible association between RBCT and nosocomial infections.

\section{MATERIAL AND METHODS}

A prospective cohort study was conducted in patients younger than 15 years old with one or more body organ systems at risk of severe functional sequelae or death. They were admitted to Hospital Universitario de Pediatría "Dr. Agustín Zubillaga", located in the city of Barquisimeto, Venezuela, and followed up until their death, transfer or discharge. They were hospitalized in the combined medical and surgical department between February 1 ${ }^{\text {st }}, 2012$ and February 1 ${ }^{\text {st }}, 2014$.

Children who died within 24 hours of admission, developed sepsis or pneumonia 48 hours before or after hospitalization, had confirmed immunodeficiency, burn wounds, long-term catheters, those whose body fluid specimens were suspected to be contaminated and those who received chemotherapy before or after their hospitalization were excluded.

All eligible patients were monitored for nosocomial infection. A nosocomial infection was considered to be post-transfusional if it occurred within 14 days after RBCT. In the group that did not receive a RBCT, nosocomial infections were recorded if they occurred during their hospital stay. An infection occurring within 48 hours before or after hospitalization was not considered nosocomial. A transfusion included the administration of one or more units of nonleukocyte-depleted packed red blood cells. 
For non-transfused subjects, the baseline hemoglobin value was that obtained at the time of admission; for transfused subjects, it was that recorded before the transfusion.

For subjects who received more than one transfusion, baseline hemoglobin was recorded as the average of hemoglobin levels before each transfusion.

The software used for statistical analysis was SPSS version 17.0. Mann-Whitney $U$ test and Student's $t$ test were used as required; differences in categorical outcome measures were analyzed using a $\chi^{2}$ test. The relevant outcome measures resulting from the univariate analysis were used to develop a logistic regression model with a $p$ value below 0.05 as an inclusion criterion. The "input" method was used.

\section{RESULTS}

One hundred and sixty two patients were included in this study. Of them, 49 (30.2\%) received a RBCT and 35 (21.6\%) developed a nosocomial infection. Baseline characteristics of transfused and non-transfused children are summarized in Table 1. Among the 113 non-transfused patients, 12 had a nosocomial infection, while among the 49 patients who received a transfusion, 23 developed an infection. This evidences a significantly higher proportion in this group $(46.9 \%$ versus $10.6 \%, p<0.0001)$. Also in this group, there was a significantly higher rate of bloodstream infections ( $18.4 \%$ versus $5.3 \%$, $p=0.008)$, sepsis $(16.3 \%$ versus $1.8 \%, p=0.0004)$, and ventilator-associated pneumonia $(10.2 \%$ versus $0.9 \%, p=0.004)$, in addition to a higher mortality rate $(28.6 \%$ versus $12.4 \%, p=0.012)$.

Table 2 describes the univariate analysis of potential risk factors for nosocomial infection. Patients with nosocomial infection had a longer hospital length of stay $(13.2 \pm 7.9$ versus $6.7 \pm 2.5$ days, OR: $1.3,95 \%$ CI: 1.2-1.5, $p<0.0001)$, a higher proportion of mechanical ventilation requirements (82.9\% versus $48.8 \%$, OR: $4.7,95 \%$ CI: $1.8-12.2$, $p=0.001)$ and a higher number of days on mechanical ventilation $(7.2 \pm 5.0$ versus $1.9 \pm 2.2$ days, OR: 1.6, 95\% CI: 1.3-1.8, $p<0.0001)$. Among patients with nosocomial infection, a higher proportion of catheter use $(82.9 \%$ versus $60.6 \%$, OR: 2.9, 95\% CI: 1.2-7.6, $p=0.022$ ), parenteral nutrition (80.0\% versus $26.0 \%$, OR: $10.4,95 \%$ CI: $4.1-26.1$, $p<0.0001$ ), RBCT ( $48.5 \%$ versus $14.9 \%$, OR: $5.4,95 \%$ CI: $2.4-12.6, p<0.0001)$, three or more RBCTs $(31.4 \%$

TABLE 1. Study population characteristics based on transfusion type

\begin{tabular}{|c|c|c|c|c|}
\hline & All & RBCT & Non-RBCT & $p$ \\
\hline Number of patients & 162 & 49 & 113 & ------- \\
\hline Age (years) & $7.1 \pm 3.9$ & $9.1 \pm 4.1$ & $6.6 \pm 3.7$ & $0.001^{*}$ \\
\hline Male sex (\%) & $80(49.4)$ & $38(77.6)$ & $42(37.2)$ & $<0.0001$ \\
\hline PRISM (score) & $13.9 \pm 3.2$ & $14.2 \pm 2.7$ & $14.1 \pm 0.4$ & $0.239 *$ \\
\hline Length of hospital stay (days) & $8.0 \pm 4.9$ & $10.5 \pm 2.3$ & $7.3 \pm 5.2$ & $<0.0001^{£}$ \\
\hline Mechanical ventilation (\%) & $90(55.6)$ & $40(81.6)$ & $50(44.2)$ & $<0.0001$ \\
\hline Days on mechanical ventilation & $2.9 \pm 3.7$ & $6.3 \pm 1.1$ & $2.1 \pm 3.6$ & $<0.0001^{*}$ \\
\hline Catheters§ $(\%)$ & $105(64.8)$ & $38(77.6)$ & $67(59.3)$ & 0.025 \\
\hline Parenteral nutrition (\%) & $60(37.0)$ & $38(77.6)$ & $22(19.5)$ & $<0.0001$ \\
\hline Nosocomial infection (\%) & 35 (21.6) & $23(46.9)$ & $12(10.6)$ & $<0.0001$ \\
\hline Bloodstream infection (\%) & $15(9.2)$ & $9(18.4)$ & $6(5.3)$ & 0.008 \\
\hline BSI (x 1000 days on CVC) & 14 & 15 & 12 & $0.061^{* *}$ \\
\hline Sepsis $(\%)$ & $10(6.2)$ & $8(16.3)$ & $2(1.8)$ & 0.0004 \\
\hline Pneumonia (\%) & $6(3.7)$ & $5(10.2)$ & $1(0.9)$ & 0.004 \\
\hline Urinary tract infection (\%) & $4(2.5)$ & $1(2.0)$ & $3(2.7)$ & 0.817 \\
\hline Surgery (\%) & $42(25.9)$ & $17(34.7)$ & $25(22.1)$ & 0.094 \\
\hline Trauma (\%) & $27(16.7)$ & $8(16.3)$ & $19(16.8)$ & 0.939 \\
\hline Complications (\%) & $74(45.7)$ & $21(42.9)$ & $53(46.9)$ & 0.635 \\
\hline Platelet and/or FFP transfusion (\%) & $88(54.3)$ & $26(53.1)$ & $62(54.9)$ & 0.895 \\
\hline Baseline hemoglobin (g/dl) & $9.6 \pm 1.9$ & $8.4 \pm 0.6$ & $9.8 \pm 2.1$ & $<0.0001^{£}$ \\
\hline Mortality (\%) & $28(17.3)$ & $14(28.6)$ & $14(12.4)$ & 0.012 \\
\hline
\end{tabular}

* Student's $t$ test; ${ }^{* *}$ Estimated by logistic regression, controlling procedure days; ${ }^{\circledR}$ Mann-Whitney U test;

RBCT: red blood cell transfusion; PRISM: pediatric risk of mortality; BSI: bloodstream infection; CVC: central venous catheter; MV: mechanical ventilation; FFP: fresh frozen plasma. ${ }^{\circledR}$ Catheters: including percutaneous catheters, phlebotomy and arterial lines, peritoneal drainage catheters, ventriculostomy and ventriculoperitoneal shunt. 
versus 5.5\%, OR: 7.6, 95\% CI: 2.6-21.9, $p<0.0001)$ and surgery (42.9\% versus $22.0 \%$, OR: $2.7,95 \%$ CI: $1.2-6.0, p=0.015)$ was observed. Mortality was also higher in this group ( $45.7 \%$ versus $10.2 \%$, OR: 7.4 , 95\% CI: 3.1-18.2, $p<0.0001$ ).

The multivariate logistic regression analysis (Table 3) shows that RBCT was independently associated with nosocomial infections (OR: 4.2, 95\% CI: 2.1-20.2, $p=0.049$ ). Other factors associated with nosocomial infections in the regression analysis included mechanical ventilation requirement (OR: 5.9, 95\% CI: 1.6-53.0, $p=0.023$ ), days on mechanical ventilation (OR: $2.8,95 \% \mathrm{CI}$ : 1.4-5.9, $p=0.003$ ), and catheter use (OR: 7.2, 95\% CI: $1.3-17.6, p=0.038)$.

\section{DISCUSSION}

Nosocomial infections are a major public health problem. In the cohort described here, RBCT was identified as an independent factor associated with nosocomial infections in settings of medical and surgical care provided to critically ill children. According to the logistic regression analysis, independent factors associated with increased risk for nosocomial infections included mechanical ventilation requirement, days on mechanical ventilation, catheter use and red blood cell transfusion. Similar data have been reported in the literature. ${ }^{6,7,8}$

Mechanical ventilation requirement and invasive catheter use are known risk factors for nosocomial infections..$^{9,10}$ The association between RBCTs and nosocomial infections is biologically possible by means of known mechanisms. . $11,12,13$ Transfusions may provide an inherent immunosuppression. Mechanisms proposed for such immunosuppression include induction of $\mathrm{T}$ cell suppression and reduced natural killer $\mathrm{T}$ cell activity. ${ }^{14}$ A reduced production of interleukin-2 and an increased production of prostaglandin E2, with a decrease in CD4 T helper cells and positive interleukin-2 receptor in helper cells. However, a

TABLE 2. Anivariate analysis: Potential risk factors for nosocomial infection

\begin{tabular}{lcccc}
\hline & Nosocomial infection & No nosocomial infection & OR (95\% CI) & $p$ \\
\hline Number of patients & 35 & 127 & & $\overline{0}$ \\
Age (years) & $8.2 \pm 4.2$ & $6.8 \pm 3.8$ & $1.1(0.9-1.2)$ & $0.088^{*}$ \\
Male sex (\%) & $17(48.5)$ & $63(49.6)$ & $1.0(0.4-2.1)$ & 0.908 \\
PRISM (score) & $15.6 \pm 3.0$ & $14.3 \pm 2.9$ & $0.9(0.3-1.8)$ & $0.137^{*}$ \\
Length of hospital stay (days) & $13.2 \pm 7.9$ & $6.7 \pm 2.5$ & $1.3(1.2-1.5)$ & $<0.0001^{*}$ \\
Mechanical ventilation (\%) & $29(82.9)$ & $62(48.8)$ & $4.7(1.8-12.2)$ & 0.001 \\
Days on mechanical ventilation & $7.2 \pm 5.0$ & $1.9 \pm 2.2$ & $1.6(1.3-1.8)$ & $<0.0001^{*}$ \\
Catheters (\%) & $29(82.9)$ & $77(60.6)$ & $2.9(1.2-7.6)$ & 0.022 \\
Parenteral nutrition (\%) & $28(80.0)$ & $33(26.0)$ & $10.4(4.1-26.1)$ & $<0.0001$ \\
RBCT (\%) & $17(48.5)$ & $19(14.9)$ & $5.4(2.4-12.6)$ & $<0.0001$ \\
Three or more RBCTs (\%) & $11(31.4)$ & $7(5.5)$ & $7.6(2.6-21.9)$ & $<0.0001$ \\
Surgery (\%) & $15(42.9)$ & $28(22.0)$ & $2.7(1.2-6.0)$ & 0.015 \\
Trauma (\%) & $5(14.3)$ & $22(17.3)$ & $0.9(0.3-2.5)$ & 0.794 \\
Mortality (\%) & $16(45.7)$ & $13(10.2)$ & $7.4(3.1-18.2)$ & $<0.0001$ \\
\hline
\end{tabular}

* Univariate regression; OR: odds ratio; CI: confidence interval; RBCT: red blood cell transfusion; PRISM: pediatric risk of mortality.

TABLE 3. Multivariate logistic regression analysis: assessment of risk for nosocomial infection

\begin{tabular}{lcccc}
\hline Outcome measure & $\beta$ & Odds ratio & 95\% confidence interval & $p$ \\
\hline Length of stay & -0.206 & 0.8 & $0.6-1.1$ & 0.231 \\
Mechanical ventilation & 3.07 & 5.9 & $1.6-53.0$ & 0.023 \\
Days on mechanical ventilation & 1.05 & 2.8 & $1.4-5.9$ & 0.003 \\
Catheters & 2.84 & 7.2 & $1.3-17.6$ & 0.038 \\
Parenteral nutrition & -0.823 & 0.5 & $0.1-7.4$ & 0.569 \\
RBCT & 0.236 & 4.2 & $2.1-20.2$ & 0.049 \\
Three or more RBCTs & -0.341 & 0.7 & $0.1-3.6$ & 0.169 \\
Surgery & -0.186 & 0.8 & $0.2-2.9$ & 0.770 \\
\hline
\end{tabular}

RBCT: red blood cell transfusion; $\beta$ : $\beta$ coefficient. The model correctly classifies $93.2 \%$ of cases. Hosmer-Lemeshow test $=0.319$. 
recent interest in immunomodulatory effects and lesions caused in relation to storage of transfused red blood cells has been documented. ${ }^{15}$

Another aspect that should be taken into consideration is whether the association observed between RBCT and nosocomial infections is an actual or a causal association given the more severe disease characteristics of patients with transfusion requirements. The pediatric risk of mortality (PRISM) was not stratified in this study; however, both children who received and did not receive a transfusion had a similar PRISM score, like children with or without a nosocomial infection. In addition, the proportion of complications and a worsening condition was similar in both groups. It may be inferred that disease severity was not an associated risk factor in the studied series, even though transfused patients had a longer hospital stay and, therefore, a longer exposure to devices.

A limitation of this study was that it did not consider an event that would serve as a cut-off point from which infections were observed in the non-transfused cohort. In addition, other nosocomial pneumonia cases in subjects with no mechanical ventilation were not considered.

\section{CONCLUSION}

The alleged effectiveness of RBCT for the management of anemia with minimal risks in critically ill children has been called into question. The association between RBCTs and nosocomial infections described in this cohort will always be vulnerable to criticism that such an association reflects physicians' tendency to transfuse the more severely ill patients. Data indicate that RBCTs are probably major contributors to the development of nosocomial infections in critically ill children.

\section{REFERENCES}

1. White M, Barron J, Gornbein J, Lin JA. Are red blood cell transfusions associated with nosocomial infections in pediatric intensive care units? Pediatr Crit Care Med 2010;11(4):464-8.

2. Istaphanous GK, Wheeler DS, Lisco SJ, Shander A. Red blood cell transfusion in critically ill children: a narrative review. Pediatr Crit Care Med 2011;12(2):174-83.

3. Armano R, Gauvin F, Ducruet T, Lacroix J. Determinants of red blood cell transfusions in a pediatric critical care unit: a prospective, descriptive epidemiological study. Crit Care Med 2005;33(11):2637-44.

4. Shorr AF, Jackson WL, Kelly KM, Fu M, et al. Transfusion practice and blood stream infections in critically ill patients. Chest 2005;127(5):1722-8.

5. HoJ, Sibbald WJ, Chin-YeeIH. Effects of storage on efficacy of red cell transfusion: when is it not safe? Crit Care Med 2003;31(12 Suppl):S687-97.

6. Raghavan M, Marik PE. Anemia, allogenic blood transfusion, and immunomodulation in the critically ill. Chest 2005;127(1):295-307.

7. Vamvakas EC, Blajchman MA. Transfusion-related immunomodulation (TRIM): an update. Blood Rev 2007;21(6):327-48.

8. Jeschke MG, Chinkes DL, Finnerty CC, Przkora R, et al. Blood transfusions are associated with increased risk for development of sepsis in severely burned pediatric patients. Crit Care Med 2007;35(2):579-83.

9. Díaz E, Lorente L, Valles J, Rello J. Neumonía asociada a la ventilación mecánica. Med Intensiva 2010;34(5):318-24.

10. Pronovost P, Needham D, Berenholtz S, Sinopoli D, et al. An intervention to decrease catheter-related bloodstream infections in the ICU. N Engl J Med 2006;355(26):2725-32.

11. Blumberg N. Deleterious clinical effects of transfusion immunomodulation: proven beyond a reasonable doubt. transfusion 2005;45(2 Suppl):33S-40S.

12. Silverboard H, Aisiku I, Martin GS, Adams M, et al. The role of acute blood transfusion in the development of acute respiratory distress syndrome in patients with severe trauma. J trauma 2005;59(3):717-23.

13. Shorr AF, Duh MS, Kelly KM, Kollef MH. Red blood cell transfusion and ventilator-associated pneumonia: a potential link? Crit Care Med 2004;32(3):666-74.

14. Bateman ST, Lacroix J, Boven K, Forbes P, et al. Anemia, blood loss, and blood transfusions in North American children in the intensive care unit. Am J Respir Crit Care Med 2008;178(1):26-33.

15. Valentine SL, Lightdale JR, Tran CM, Jiang H, et al. Assessment of hemoglobin threshold for packed RBC transfusion in a medical-surgical PICU. Pediatr Crit Care Med 2014;15(2):e89-94. 